\title{
A Multivariate Time-Frequency Approach for Tracking QT Variability Changes Unrelated to Heart Rate Variability
}

\author{
Michele Orini $^{1}$, Peter Taggart ${ }^{1}$ and Pier D Lambiase ${ }^{1}$
}

\begin{abstract}
The beat-to-beat variability of the QT interval (QTV) is a marker of ventricular repolarization (VR) dynamics and it has been suggested as an index of sympathetic ventricular outflow and cardiac instability. However, QTV is also affected by RR (or heart rate) variability (RRV), and QTV due to RRV may reduce QTV specificity as a VR marker. Therefore, it would be desirable to separate QTV due to VR dynamics from QTV due to RRV. To do that, previous work has mainly focused on heart rate corrections or time-invariant autoregressive models. This paper describes a novel framework that extends classical multiple inputs/single output theory to the time-frequency (TF) domain to quantify QTV and RRV interactions. Quadratic TF distributions and TF coherence function are utilized to separate QTV into two partial (conditioned) spectra representing QTV related and unrelated to $R R V$, and to provide an estimates of intrinsic VR dynamics. In a simulation study, a timevarying ARMA model was used to generate signals representing realistic RRV and VR dynamics with controlled instantaneous frequencies and powers. The results demonstrated that the proposed methodology is able to accurately track changes in VR dynamics, with a correlation between theoretical and estimated patterns higher than 0.88 . Data from healthy volunteers undergoing a tilt table test were analyzed and representative examples are discussed. Results show that the QTV unrelated to RRV dynamics quickly increased during orthostatic challenge.
\end{abstract}

\section{INTRODUCTION}

The interval between the onset of the Q-wave and the end of the T-wave in the ECG, so called QT, is a marker of cardiac repolarization. The beat-to-beat QT variability (QTV) conveys relevant information regarding cardiac pathophysiology. Several studies have suggested that QTV may be a marker of sympathetic ventricular outflow and cardiac instability [1], [2], [3]. However, relevant aspects of QTV analysis are still only partially understood [2]. Among them, there is the interaction between QTV and RR interval variability (RRV). QTV can be modeled as the sum of a component due to ventricular repolarization (VR) dynamics [4] and another due to RRV. The former is thought to be a marker of ventricular sympathetic activity [5] and it may have a better predictive value than QTV. Therefore, it would be desirable to disentangle these two components. Previous techniques to remove the influence of RRV from QTV have mainly utilized heart rate corrections [1], time-invariant multivariate autoregressive models [5], [6] or other modelbased approaches [7]. However, there is still a need for a methodology that provides accurate and robust estimates of the dynamic profile of QTV changes unrelated to RRV.

\footnotetext{
${ }^{1} \mathrm{M}$ Orini, P Taggart and PD Lambiase are with the Institute of Cardiovascular Science, University College London, London, United Kingdom m.orini@ucl.ac.uk
}

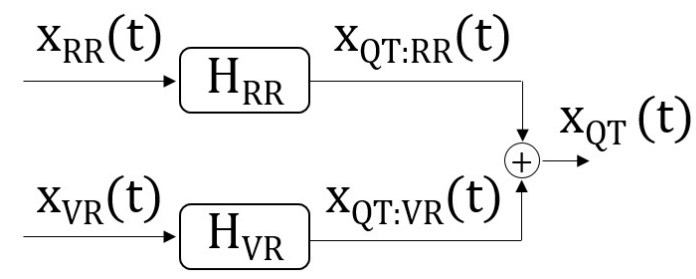

Fig. 1. Diagrammatic of the proposed model. QT variability is the sum of RR-related variability (QT:RR) and RR-unrelated variability (QT:RV) due to ventricular repolarization (VR) dynamics. $H_{\mathrm{RR}}$ and $H_{\mathrm{VR}}$ are linear transfer functions. The proposed framework uses multivariate time-frequency analysis to provide an estimates of changes in $x_{\mathrm{QT}: \mathrm{VR}}(t)$ based on measurements of $x_{\mathrm{RR}}(t)$ and $x_{\mathrm{QT}}(t)$.

The aim of this study is to propose a novel framework for estimating QTV unrelated to RRV during non-stationary conditions, that enables to specifically track changes in VR dynamics.

\section{METHOD}

\section{A. A two-inputs/One-output model}

Beat-to-beat QTV is modeled as a continuous-time signal composed of two separate contributions, $x_{\mathrm{QTTRR}}(t)$ and $x_{\mathrm{QT:VR}}(t)$ (see Fig. 1). Signal $x_{\mathrm{QTTRR}}(t)$ represents QTV due to RRV. This component includes oscillations at respiratory frequency (respiratory sinus arrhythmia) and at lower frequencies (Mayer waves). Signal $x_{\mathrm{QT:VR}}(t)$ represents oscillations unrelated to RR variability which are assumed to be due to ventricular repolarization (VR) dynamics. Mathematically, this is described as:

$x_{\mathrm{QT}}(t)=x_{\mathrm{QT}: \mathrm{RR}}(t)+x_{\mathrm{QT}: \mathrm{VR}}(t)=x_{\mathrm{RR}}(t) * h_{\mathrm{RR}}(t)+x_{\mathrm{VR}}(t) * h_{\mathrm{VR}}(t)$

where $*$ represents temporal convolution and $h_{\mathrm{RR}}(t)$ and $h_{\mathrm{VR}}(t)$ are time-varying linear filters. The proposed methodology utilizes measurements of $x_{\mathrm{QT}}(t)$ and $x_{\mathrm{RR}}(t)$ to estimate the time-frequency (TF) distribution of $x_{\mathrm{QT:VR}}(t)$.

The following formulation is an extension to the $\mathrm{TF}$ domain of classical signal processing theory describing multiple inputs/single output relationships [8]. Assuming that inputs $x_{\mathrm{RR}}(t)$ and $x_{\mathrm{RV}}(t)$ are locally uncorrelated, the transfer function $H_{\mathrm{RR}}(t, f)$, which describes $h_{\mathrm{RR}}(t)$ in the TF domain, is [8]:

$$
\left|H_{\mathrm{RR}}(t, f)\right|^{2}=\frac{S_{\mathrm{QT}: \mathrm{RR}}(t, f)}{S_{\mathrm{RR}}(t, f)}=\left|\gamma_{Q T, R R}(t, f)\right|^{2} \frac{S_{\mathrm{QT}}(t, f)}{S_{\mathrm{RR}}(t, f)}
$$

where $S_{\mathrm{x}}(t, f)$ represents the TF spectrum of a given process $x$, and $\gamma_{Q T, R R}(t, f)$ is the coherence function between $x_{\mathrm{QT}}(t)$ 
and $x_{\mathrm{RR}}(t)$. These expressions are used to estimate the spectrum of $x_{\mathrm{QT}: \mathrm{VR}}(t)$ :

$$
\begin{aligned}
S_{\mathrm{QT}: \mathrm{VR}}(t, f) & =S_{\mathrm{QT}}(t, f)-S_{\mathrm{QT}: \mathrm{RR}}(t, f) \\
& =S_{\mathrm{QT}}(t, f)-\left|H_{\mathrm{RR}}(t, f)\right|^{2} S_{\mathrm{RR}}(t, f) \\
& =\left(1-\left|\gamma_{R R, Q T}(t, f)\right|^{2}\right) S_{\mathrm{QT}}(t, f)
\end{aligned}
$$

and the spectrum of $x_{\mathrm{QT}: \mathrm{RR}}(t)$ :

$$
S_{\mathrm{QT}: \mathrm{RR}}(t, f)=\left|\gamma_{R R, Q T}(t, f)\right|^{2} S_{\mathrm{QT}}(t, f)
$$

\section{B. Time-frequency analysis}

Time-frequency spectra and coherence in (2) were estimated using the framework proposed in [9], [10] based on Cohen's class distributions. Considering two continuous-time signals, $x_{i}(t)$ and $x_{k}(t)$, spectra are defined as:

$$
S_{i, k}(t, f)=\iint_{-\infty}^{\infty} \phi_{\mathrm{d}-\mathrm{D}}(\tau, v) A_{i, k}(\tau, v) e^{j 2 \pi(t v-f \tau)} d v d \tau
$$

where $A_{i, k}(\tau, v)$ is the ambiguity function of $x_{i}(t)$ and $x_{k}(t)$, and $\phi_{\mathrm{d}-\mathrm{D}}(\tau, v)$ is the elliptical exponential kernel defined in [9]. The TF coherence is estimated as [9]:

$$
\gamma_{i, j}(t, f)=\frac{S_{i, k}(t, f)}{\sqrt{S_{i, i}(t, f) S_{k, k}(t, f)}} ;\left|\gamma_{i, k}(t, f)\right| \in[0,1]
$$

and it is equal to one in TF regions where the signals are linearly coupled and zero where they are uncorrelated. Time and frequency resolutions are quantified as described in [9] and were equal to $12.5 \mathrm{~s}$ and $0.039 \mathrm{~Hz}$, respectively.

\section{Experimental Setting}

The cardiovascular response to orthostatic challenge was studied in 16 healthy volunteers (aged 29 \pm 3 years) using a tilt table test. The protocol included early supine (ES) position (4 min), head-up tilt to an angle of $70^{\circ}(5 \mathrm{~min})$, and late supine (LS) position (4 min) as described in other studies [9], [11]. 12-Lead ECG was recorded with a sampling frequency of $1000 \mathrm{~Hz}$.

\section{Data Analysis}

Customized algorithms were used to detect the temporal occurrence of R-waves and T-end [12]. The latter was defined utilizing a tangent method. The QT interval was approximated by the interval from the R-wave to the end of the T-wave in lead V4. Ectopic beats and artifacts were rare. When present, they were removed and the time series were interpolated. RR and QT time series were interpolated at a sampling frequency of $4 \mathrm{~Hz}$, and the RRV and QTV signals, $x_{\mathrm{RR}}(t)$ and $x_{\mathrm{QT}}(t)$, were obtained by high-pass filtering these interpolated series with a cut-off frequency of $0.03 \mathrm{~Hz}$. Instantaneous powers and coherence in a given spectral band $\Omega$ were estimated as:

$$
P_{i}^{\Omega}(t)=\int_{\Omega} S_{i, i}(t, f) d f ; \quad \gamma_{i, k}^{\Omega}(t)=\int_{\Omega} \gamma_{i, k}(t, f) d f
$$

where $\Omega$ includes the following spectral bands: $L F \in[0.03-$ $0.15] \mathrm{Hz}, H F \in[0.15-0.50] \mathrm{Hz}$ and $T O T \in[0.03-1.00]$
Hz. Relative changes of $P_{\mathrm{QT}: \mathrm{VR}}^{\Omega}(t)$ during tilt with respect to baseline were measured as:

$$
R_{\Omega}=\operatorname{mean}\left(P_{\mathrm{QT}: \mathrm{VR}}^{\Omega}(t)\right)_{t \in I} / \operatorname{mean}\left(P_{\mathrm{QT}: \mathrm{VR}}^{\Omega}(t)\right)_{t \in B L}
$$

where BL and I are intervals that goes from 1 min after the beginning of early supine and tilt to $1 \mathrm{~min}$ before the end of early supine and tilt, respectively.

\section{E. Simulation Study}
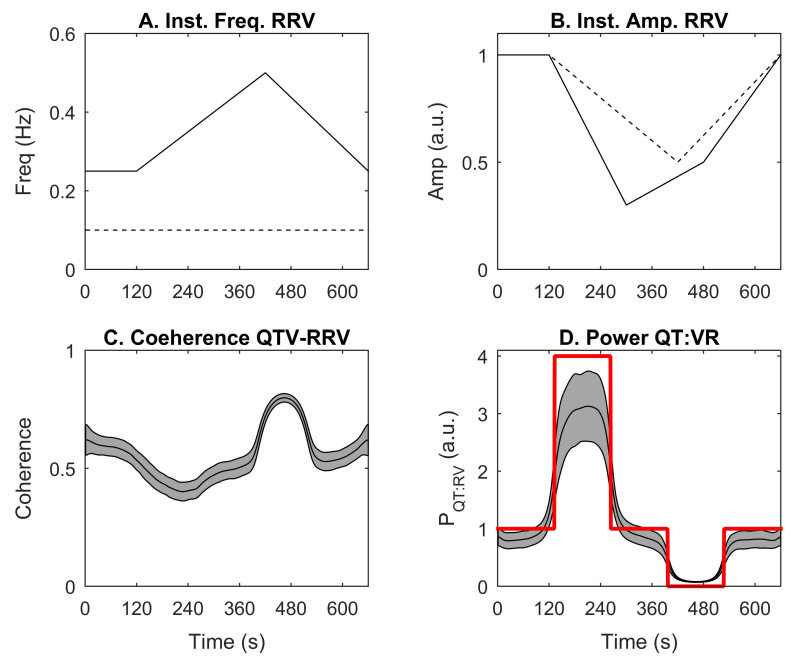

Fig. 2. Simulation study. A and B: Instantaneous frequencies and amplitude of $x_{\mathrm{RR}}(t)$. Solid and dashed lines represent HF and LF, respectively. C: Coherence $\gamma_{\mathrm{QT}, \mathrm{RR}}^{\mathrm{TOT}}(t)$ (mean $\pm \mathrm{sd}$ ). D: Instantaneous power of QTV due to ventricular repolarization $(\mathrm{VR}), P_{\mathrm{QT}: \mathrm{VR}}^{\mathrm{TOT}}(t)$ from the model (red) and estimated with the proposed methodology (black, mean $\pm \mathrm{sd}$ ).

The methodology was tested in challenging conditions, mimicking RRV and VRV during exercise stress testing. The model shown in Fig. 1 was used to generate signals with controlled dynamics. The signal representing RRV, $x_{\mathrm{RR}}(t)$, was the sum of a AM LF and an AM-FM HF component (see Fig. 2A-B), and it was generated using the time-varying ARMA scheme described in [13]. The signal representing $\mathrm{VR}, x_{\mathrm{VR}}(t)$, was a white Gaussian noise with amplitude modulation associated with the instantaneous power, $P_{\mathrm{QT}: \mathrm{VR}}^{\mathrm{TOT}}(t)$, shown as a red solid line in Fig. 2D. Transfer functions were assumed to be scaling factors, $H_{\mathrm{RR}}(t, f)=H_{\mathrm{VR}}(t, f)=$ $0.20 / \sigma_{\mathrm{RR}}$, where $\sigma_{\mathrm{RR}}$ is the standard deviation of $x_{\mathrm{RR}}(t)$. The correlation coefficient between theoretical and estimated $P_{\mathrm{QT}: \mathrm{VR}}^{\mathrm{TOT}}(t)$ was calculated to assess the proposed methodology.

\section{RESULTS}

\section{A. Results of the Simulation Study}

Figure 2D shows that the proposed model provided accurate estimates of $P_{\mathrm{QT}: \mathrm{VR}}^{\mathrm{TOT}}(t)$. Pearson's and Sperman's correlation coefficients between theoretical and estimated $P_{\mathrm{QT}: \mathrm{VR}}^{\mathrm{TOT}}(t)$ were $0.888 \pm 0.021$ and $0.873 \pm 0.002$, respectively $(\mathrm{N}=500$ iterations). 

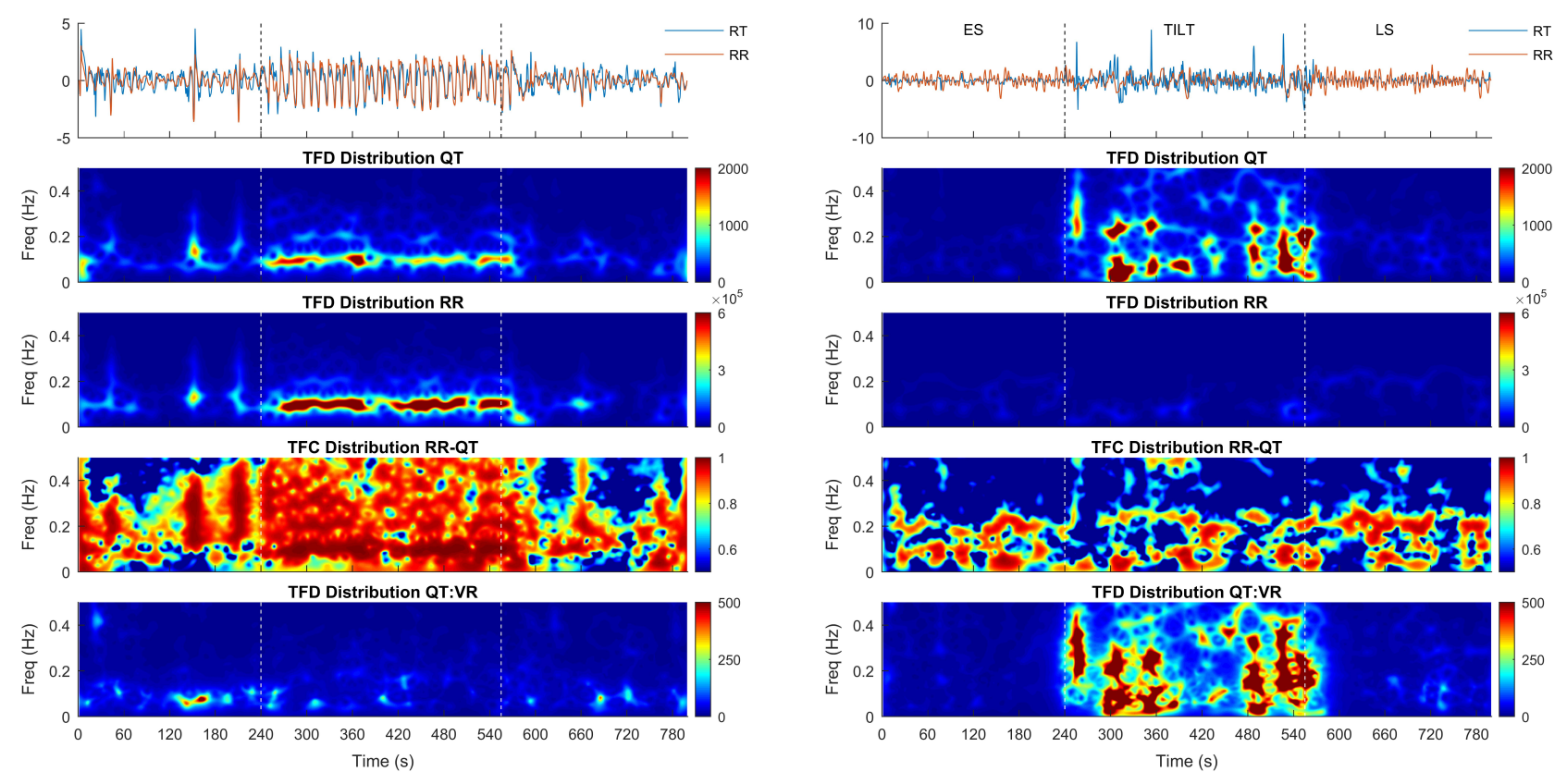

Fig. 3. Results from one subject showing QTV mainly related to RRV (left) and poorly related to RRV (right). From top to bottom: QT and RR variability normalized by their standard deviation. Time-frequency distributions of QTV and RRV. Time-frequency coherence between QTV and RRV. TF distribution of $x_{\mathrm{QT}: \mathrm{VR}}(t)$, i.e. QTV unrelated to RRV.

\section{B. Real Data}

Examples of spectra of $x_{\mathrm{QT}}(t), x_{\mathrm{RR}}(t)$ and $x_{\mathrm{QT}: \mathrm{VR}}(t)$ are given in Fig. 3. Panels on the left are representative of a subject whose QTV is mainly driven by RRV. Spectra $S_{\mathrm{QT}}(t, f)$ and $S_{\mathrm{RR}}(t, f)$ present similar features and are characterized by high TF coherence. As a result, RR-unrelated QTV $S_{\text {QT:VR }}(t, f)$ is low. In particular, the increase in the LF spectral band of $S_{\mathrm{QT}}(t, f)$ observed during head-up tilt is mainly driven by $S_{\mathrm{RR}}(t, f)$ and therefore it does not appear in $S_{\mathrm{QT:VR}}(t, f)$. Panels on the right show an example where QTV is largely uncorrelated to RRV. $S_{\mathrm{QT}}(t, f)$ is low in supine position and it increases during tilt. The temporal and spectral organization of QTV and RRV do not correlate, and $\gamma_{\mathrm{RR}, \mathrm{QT}}(t, f)$ is lower than 0.5 in a large proportion of the TF domain. As a result, $S_{\mathrm{QT:VR}}(t, f)$ is similar to $S_{\mathrm{QT}}(t, f)$. The comparison between the instantaneous power and coherence from these two examples is displayed in Fig. 4. Results from the subject showing high and low coherence between QTV and RRV are reported on the left and right panels, respectively. In both cases $P_{\mathrm{OT}: \mathrm{VR}}^{\mathrm{TOT}}(t)<P_{\mathrm{QT}}^{\mathrm{TOT}}(t)$, and the degree of similarity between them is determined by $\gamma_{\mathrm{QT}, \mathrm{RR}}(t, f)$. Figure 5 shows the relation between $\gamma_{\mathrm{QT}, \mathrm{RR}}(t)$, whose temporal mean is represented on the horizontal axis, and the correlation coefficient between $P_{\mathrm{QT}}^{\mathrm{TOT}}(t)$ and $P_{\mathrm{QT}: \mathrm{VR}}^{\mathrm{TOT}}(t)$ across all subjects $(\mathrm{n}=16)$. The highly negative correlation $(c=-0.83)$ means that the higher $\gamma_{\mathrm{QT}, \mathrm{RR}}(t)$ the lower the similarity between $P_{\mathrm{QT}}^{\mathrm{TOT}}(t)$ and $P_{\mathrm{QT}: \mathrm{VR}}^{\mathrm{TOT}}(t)$. Therefore, this methodology modifies the time course of QTV and its two components based on local TF coupling, and the resulting correction is time-varying, data-driven and subject-specific. The change in spectral power during tilt with respect to baseline was equal to $R_{\mathrm{Tilt}}^{\mathrm{LF}}=4.17 \pm 1.39, R_{\mathrm{Tilt}}^{\mathrm{HF}}=5.13 \pm 1.44$,
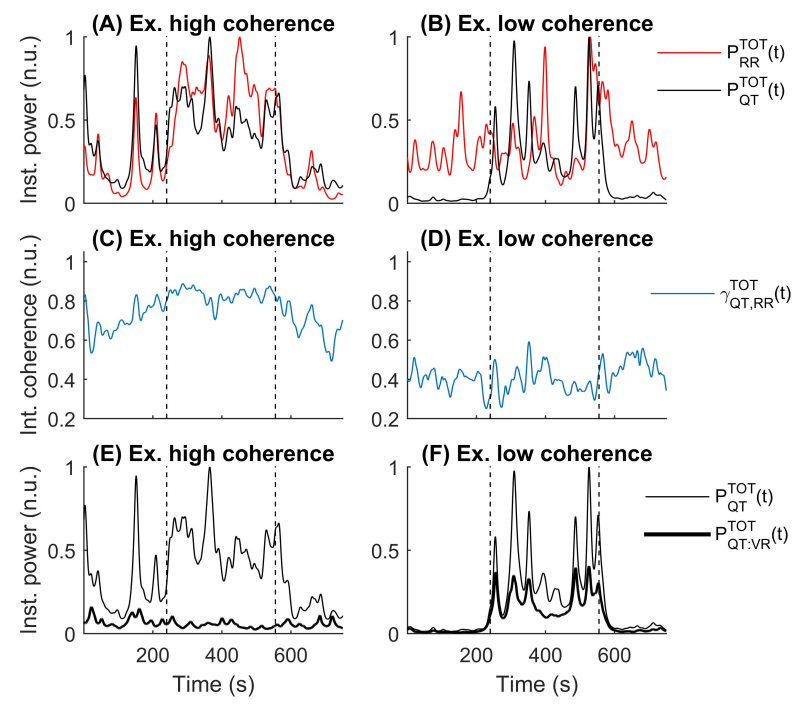

Fig. 4. Comparison between the instantaneous power and coherence from the examples shown in Fig. 3. When coherence between QTV and RRV is high (or low), instantaneous power of QTV and QTV unrelated to RRV has a similar (o different) pattern.

$R_{\text {Titt }}^{\mathrm{TOT}}=4.65 \pm 1.31$. All changes were statistically significant $(P<0.05$, Wilcoxon signed-rank test $)$.

\section{DISCUSSION}

A new method to track changes in QTV unrelated to RRV during non-stationary conditions is proposed. The results of the simulation study demonstrates that it provides accurate estimates of QTV unrelated to RRV, with correlation with theoretical variability higher than 0.88 . The analysis of data from healthy volunteers shows that QTV unrelated to RRV 


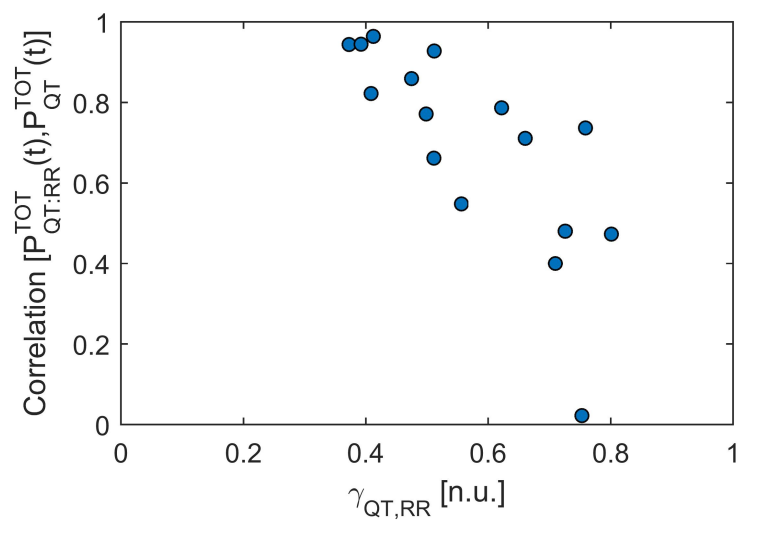

Fig. 5. Relation between the mean QTV-RRV TF coherence (horizontal axis) and the degree of similarity between the time course of instantaneous powers of QTV and QTV unrelated to RRV. The higher the coherence between QTV and RRV and the more different the instantaneous powers of QTV and QTV unrelated to RRV.

increased during tilt table test.

This methodology extends classical multiple inputs/single output theory [8] to the TF domain to study the interactions between QTV and RRV. Cohen's class TF distributions and TF coherence function [9] are utilized to separate QTV into two partial TF spectra [10], [14], representing QTV related and unrelated to RRV. To the best of our knowledge, this is the first work presenting a non-stationary model-free methodology to disentangle changes in RRV related and RRV unrelated QTV. In this framework, QTV unrelated to RRV is assumed to be due to ventricular repolarization (VR) dynamics, and the two inputs, RR and VR variability, are assumed to be uncorrelated. This assumption is based on the fact that RR and VR variability have different anatomical origins, i.e. the sinus node and the ventricular myocardium. However, common drivers cannot be excluded since respiratory and low-frequency oscillations have been observed in human VR dynamics [15], [16] and both RRV and QTV are known to be affected by sympathetic modulation [2]. Of note, this methodology allows $x_{\mathrm{QT:VR}}(t)$ and $x_{\mathrm{QT}: \mathrm{RR}}(t)$ to have components in the same $\mathrm{TF}$ regions, since their power is assigned from QTV proportionally to the squared magnitude of the TF coherence function. The framework used in the simulation study tested the proposed methodology in a challenging situation: signals were AM-FM random processes with quick changes in instantaneous frequency and amplitude, and VR dynamics was a white Gaussian noise with fast amplitude modulation. Nevertheless, correlation between theoretical and estimated instantaneous QT:RV power was very high. However, since TF coherence is a biased estimate [9] the estimates were also slightly biased (see Fig. 2D).

These promising results may be the basis for further study to assess the role of this methodology as a marker of ventricular sympathetic activity and cardiac instability.

\section{ACKNOWLEDGMENT}

MO was supported by a Marie Curie IEF-2013 fellowship.

\section{REFERENCES}

[1] M. N. Niemeijer, M. E. van den Berg, M. Eijgelsheim, G. van Herpen, B. H. Stricker, J. A. Kors, and P. R. Rijnbeek, "Short-term qt variability markers for the prediction of ventricular arrhythmias and sudden cardiac death: a systematic review," Heart, 2014.

[2] M. Baumert, A. Porta, M. A. Vos, M. Malik, J.-P. Couderc, P. Laguna, G. Piccirillo, G. L. Smith, L. G. Tereshchenko, and P. G. Volders, "Qt interval variability in body surface ecg: measurement, physiological basis, and clinical value: position statement and consensus guidance endorsed by the european heart rhythm association jointly with the esc working group on cardiac cellular electrop," Europace, pp. -, Jan. 2016.

[3] L. G. Tereshchenko, B. J. Fetics, P. P. Domitrovich, B. D. Lindsay, and R. D. Berger, "Prediction of ventricular tachyarrhythmias by intracardiac repolarization variability analysis," Circulation: Arrhythmia and Electrophysiology, vol. 2, no. 3, pp. 276-284, 2009.

[4] E. Pueyo, A. Corrias, L. Virg, N. Jost, T. Szl, A. Varr, N. Szentandrssy, P. P. Nnsi, K. Burrage, and B. Rodrguez, "A multiscale investigation of repolarization variability and its role in cardiac arrhythmogenesis." Biophys. J., vol. 101, no. 12, pp. 2892-2902, Dec 2011.

[5] A. Porta, E. Tobaldini, T. Gnecchi-Ruscone, and N. Montano, "Rt variability unrelated to heart period and respiration progressively increases during graded head-up tilt," American Journal of Physiology - Heart and Circulatory Physiology, vol. 298, no. 5, pp. H1406H1414, 2010.

[6] R. Almeida, S. Gouveia, A. Rocha, E. Pueyo, J. Martinez, and P. Laguna, "Qt variability and hrv interactions in ecg: Quantification and reliability," IEEE Trans. Biomed. Eng., vol. 53, no. 7, pp. 1317-1329, 2006.

[7] G. Valenza, M. Orini, L. Citi, A. Minchole, E. Pueyo, P. Laguna, and R. Barbieri, "Assessing real-time rr-qt frequency-domain measures of coupling and causality through inhomogeneous point-process bivariate models." Conf Proc IEEE Eng Med Biol Soc, vol. 2014, pp. 6475-6478, 2014.

[8] J. S. Bendat and A. G. Piersol, "Multiple-input/output relationships," in Random Data. John Wiley \& Sons, Inc., 2012, pp. 201-247.

[9] M. Orini, R. Bailon, L. T. Mainardi, P. Laguna, and P. Flandrin, "Characterization of dynamic interactions between cardiovascular signals by time-frequency coherence." IEEE Trans. Biomed. Eng., vol. 59, no. 3, pp. 663-673, Mar 2012.

[10] M. Orini, R. Bailon, P. Laguna, L. Mainardi, and R. Barbieri, "A multivariate time-frequency method to characterize the influence of respiration over heart period and arterial pressure," EURASIP Journal on Advances in Signal Processing, vol. 2012, no. 1, p. 214, 2012.

[11] M. Orini, P. Laguna, L. T. Mainardi, and R. Bailn, "Assessment of the dynamic interactions between heart rate and arterial pressure by the cross time-frequency analysis." Physiol. Meas., vol. 33, no. 3, pp. 315-331, Mar 2012.

[12] P. Taggart, M. Orini, B. Hanson, M. Hayward, R. Clayton, H. Dobrzynski, J. Yanni, M. Boyett, and P. D. Lambiase, "Developing a novel comprehensive framework for the investigation of cellular and whole heart electrophysiology in the in situ human heart: Historical perspectives, current progress and future prospects," Prog. Biophys. Mol. Biol., vol. 115, no. 23, pp. 252-260, Aug. 2014.

[13] M. Orini, R. Bailón, L. Mainardi, and P. Laguna, "Synthesis of hrv signals characterized by predetermined time-frequency structure by means of time-varying arma models," Biomedical Signal Processing and Control, vol. 7, no. 2, pp. 141 - 150, 2012.

[14] D. Widjaja, M. Orini, E. Vlemincx, and S. Van Huffel, "Cardiorespiratory dynamic response to mental stress: A multivariate time-frequency analysis," Computational and Mathematical Methods in Medicine, vol. 2013, p. 12, 2013.

[15] B. Hanson, N. Child, S. Van Duijvenboden, M. Orini, Z. Chen, R. Coronel, C. A. Rinaldi, J. S. Gill, J. S. Gill, and P. Taggart, "Oscillatory behavior of ventricular action potential duration in heart failure patients at respiratory rate and low frequency." Front Physiol, vol. 5, p. 414, 2014.

[16] S. van Duijvenboden, B. Hanson, N. Child, M. Orini, C. A. Rinaldi, J. S. Gill, and P. Taggart, "Effect of autonomic blocking agents on the respiratory-related oscillations of ventricular action potential duration in humans." Am J Physiol Heart Circ Physiol, vol. 309, no. 12, pp. H2108-H2117, Dec 2015. 